\author{
Art Museums as Institutions for Flourishing \\ Katherine N. Cotter and James O. Pawelski \\ Positive Psychology Center \\ University of Pennsylvania
}

Author Note

Katherine N. Cotter and James O. Pawelski, Positive Psychology Center, University of Pennsylvania.

The opinions expressed are those of the authors and do not necessarily represent the views of the National Endowment for the Arts Office of Research \& Analysis or the National Endowment for the Arts. The National Endowment for the Arts does not guarantee the accuracy or completeness of the information included in this material and is not responsible for any consequences of its use. This NEA Research Lab is supported in part by an award from the National Endowment for the Arts (Award\#: 1862782-38-C-20). This publication was made possible through the support of a grant from Templeton Religion Trust. The opinions expressed in this publication are those of the authors and do not necessarily reflect the views of Templeton Religion Trust.

Correspondence should be addressed to Katherine N. Cotter, Positive Psychology Center, University of Pennsylvania, Philadelphia, PA, USA, email: katherinencotter@gmail.com 


\begin{abstract}
Visiting art museums is a common activity that a wide variety of people choose to engage in for many reasons. Increasingly, communities, nations, and societies are turning to art museums as institutions to contribute to flourishing (i.e., reducing ill-being factors, such as depression, and increasing well-being factors, such as feelings of belonging). In this paper, we review the existing psychological literature examining art museum visitation and museum program participation and their associations with flourishing-related outcomes. The literature suggests art museum visitation is associated with reductions in ill-being outcomes and increases in well-being outcomes. Additionally, programs targeting flourishing outcomes in clinical or at-risk populations (e.g., people living with dementia, older adults) show benefits to participants, with visits to art museums being socially prescribed across the globe to address a variety of ill-being conditions. Implications for existing knowledge and avenues for future research are discussed.
\end{abstract}

Keywords: Art museums, visual art, flourishing, well-being, ill-being, health 


\section{Art Museums as Institutions for Flourishing}

The arts and humanities help us weather the storms of life, but more than that, they can help guide us to new possibilities as we imagine and create more fulfilling lives and thriving communities.

- $\quad$ Martin E. P. Seligman, ${ }^{1}$ University of Pennsylvania

I am convinced that in the twenty-first century, culture will be to health what sports were in the twentieth century: cultural experiences will be seen to contribute to our well-being the same way sports do to our physical conditioning.

- $\quad$ Nathalie Bondil, Director of Museum and Exhibitions, Institut du Monde Arabe, Paris

Museums, libraries, and cultural institutions provide opportunities for people to understand and celebrate who they are, were, and might be.

- Jeffrey K. Smith, The Museum Effect

In 2019, over 60 million people visited the 10 most popular art museums in the world (Sharpe \& da Silva, 2020). Visiting art museums is a common activity that a wide variety of people choose to engage in (National Endowments for the Arts, 2013, 2018). People visit art museums for a variety of reasons (Cotter et al., in press; Falk, 2006, 2008; Pekarik \& Schreiber, 2012; Phelan et al., 2018), such as wanting to learn something, because they are curious about what art the

\footnotetext{
${ }^{1}$ We are honored to be able to include this article in a special issue of the Journal of Positive Psychology dedicated to Marty Seligman on the occasion of his $80^{\text {th }}$ birthday. Hearing Marty describe positive psychology in the fall of 2000 at the first public meeting for this new field inspired James Pawelski to think of his work as lying in the domain of the Positive Humanities. We offer this paper as a step forward in the work of the Positive Humanities and in profound gratitude for Marty's vision, example, and support for more than two decades.
} 
museum has on display, or to check off a "must-see" spot when on vacation. But art museum visitation also has been associated with a range of outcomes supportive of human flourishing, such as increased subjective well-being (e.g., Grossi et al., 2019; Thomson et al., 2018), higher quality of life (e.g., Schall et al., 2018; Wilkinson et al., 2007), and decreases in anxiety and depression (e.g., Binnie, 2010; D’Cunha et a., 2019). Increasingly, communities, nations, and societies are turning to art museums as institutions to foster health and well-being. In a 2019 report (OECD \& ICOM, 2019), the OECD argues that promoting health and well-being among visitors should be a central goal of art museums, and doctors in several countries can now prescribe visits to the art museum as treatment for a range of health-related conditions (e.g., All-Party Parliamentary Group, 2017; Mercer, 2018). Given the growing evidence supporting the idea that art museums can contribute to health and well-being, the purpose of this review is to consolidate research examining the link between art museum engagement and health and well-being, and what is currently known about this link in both clinical and non-clinical populations.

\section{Well-Being, Ill-Being, and Flourishing}

'Health' or 'well-being' are broad terms often used to describe a wide range of experiences and conditions related to quality of life. For example, the World Health Organization (WHO; 1948) emphasizes that health and well-being is "a state of complete physical, mental, and social wellbeing and not merely the absence of disease or infirmity." Within this definition, the WHO identifies three domains of life as the core factors making up a person's health and well-being and further emphasizes that focusing solely on illness as the primary indicator of someone's health and well-being paints an incomplete picture. Because the notion of health and well-being-for the purpose of this review collectively referred to as 'flourishing' - is a complex and multi-faceted one, breaking it down into smaller, more specific components can provide greater clarity. 
An obvious component is a focus on the presence or absence of diseases, disorders, and negative states - for the purpose of this review, this component of flourishing will be referred to as 'ill-being'. Ultimately the goal of addressing ill-being is to mitigate and eliminate negative experiences and to prevent the future occurrence of these negative experiences. Ill-being is often the focus within current health systems with people seeking out a doctor or clinician when they are feeling unwell (i.e., mitigation and elimination) or completing routine check-ups to identify warning signs of future ill-being (i.e., prevention). In psychology, an ill-being focus would include examination of existing psychological disorders or symptoms of disorders, negative emotions, or stressors and the prevention of such conditions. Certainly, reducing impacts of ill-being and preventing future ill-being are important factors in being 'well', but they are not the only factors to be considered.

A complementary component to ill-being is a focus on the cultivation of strengths, meaning, and positive states and traits-for the purpose of this review, this component of flourishing will be referred to as 'well-being'. Within a health context, well-being may be seen as practicing positive health behaviors, such as having adequate quality and quantity of sleep, eating a sufficient diet, or exercising regularly. Although these behaviors — to some extent—may buffer against ill-being conditions, they also result in positive states in addition to some preventative functions. Within psychology, well-being is often studied under the umbrella of positive psychology (Seligman, 2012; Seligman \& Csikszentmihalyi, 2000). Positive psychology emphasizes the promotion of positive experiences and qualities and seeks to preserve these experiences and qualities. Theories and discussions of well-being have identified a variety of components that contribute to well-being, such as experiencing positive emotions (Diener et al., 1999; Fredrickson, 1998; Seligman, 2012), engagement in life and activities (Csikszentmihalyi, 
1990; Ryff, 1995; Ryff \& Singer, 1998; Seligman, 2012), having positive relationships (Prilleltensky et al., 2015; Ryan \& Deci, 2000; Ryff, 1995; Ryff \& Singer, 1998; Seligman, 2012; VanderWeele, 2017), cultivating positive traits and character strengths (Niemiec, 2013; Peterson \& Seligman, 2004; VanderWeele, 2017), having interesting and perspective-changing experiences (Oishi \& Westgate, 2021), feeling a meaning or purpose in life (Ryff, 1995; Ryff \& Singer, 1998; Seligman, 2012; VanderWeele, 2017), having financial and material stability (VanderWeele, 2017), having autonomy (Ryan \& Deci, 2000; Ryff, 1995; Ryff \& Singer, 1998), and feelings of achievement or competence (Ryan \& Deci, 2000; Seligman, 2012). Well-being oriented approaches, in contrast to ill-being's emphasis on prevention and mitigation, seek to promote new positive outcomes and preserve existing positive outcomes (Pawelski, 2016a).

Viewing ill-being and well-being as constituent elements of overall flourishing-with flourishing referring to ill-being and well-being collectively-provides greater specificity when discussing research findings and aids in identifying gaps in the literature (Gable \& Haidt, 2005; Pawelski, 2013). For instance, ill-being research often focuses on individuals with clinical diagnoses, at risk for clinical diagnosis, or who otherwise face hardships in their lives (e.g., members of minority groups, Close et al., 2016; workers in high-stress occupations, Sargent et al., 2017). Intervention and measurement strategies emphasize the ability to mitigate or prevent illbeing related experiences and less frequently also consider impacts on well-being. Conversely, well-being research often emphasizes non-clinical populations or populations with unique positive characteristics (e.g., West Point cadets or National Spelling Bee competitors, Duckworth et al., 2007) with intervention and measurement strategies emphasizing improvements in well-being related outcomes over reductions in ill-being related outcomes. 
Given their respective foci, it is logical that ill-being and well-being approaches to flourishing would emphasize certain constructs over others, yet it is vital to recognize that ill-being and well-being co-exist: The presence of ill-being does not preclude the ability to experience wellbeing and experiencing well-being does not imply the absence of ill-being. Likewise, clinical populations are not devoid of well-being experiences and non-clinical populations will experience ill-being. It is also possible to experience ill-being in some life domains (e.g., personal relationships) while simultaneously experiencing well-being in other domains (e.g., work life). Although well-being and ill-being are inversely related (e.g., Davidson, 1998; Diener et al., 1995; Fava et al., 2004; Keyes, 2002, 2007; Russell \& Carroll, 1999; Ryff et al., 2006; Urry et al., 2004), the weakness of the association indicates that these two components of flourishing are not opposite ends of the same spectrum; rather, they represent separable experiences that require independent study. To this end, in addition to including both ill-being and well-being when discussing flourishing, it is necessary to consider these components of flourishing in both clinical and nonclinical populations and in individuals who are languishing or thriving (see Keyes, 2002, 2007 for additional discussion).

In sum, the study of flourishing requires an understanding of the impact of both mitigating and preventing ill-being and of promoting and preserving well-being (Pawelski, 2016a). Since well-intended efforts can sometimes have unintended negative consequences, it can be useful to employ a eudaimonic profile (Pawelski, 2016b) for a comprehensive analysis of factors supporting and reducing flourishing. Although it can be useful to investigate each of these factors separately, it is also important to be clear on how they interact. Flourishing may be supported (or in some ways, thwarted) by activities and practices that target ill-being or well-being - or both. These activities and practices may come in the form of engaging in standardized, targeted treatment 
protocols administered by clinical professionals (e.g., beginning psychotherapy), self-initiated behavior or activity changes (e.g., enrolling in a mindfulness course), or tweaks and nudges to existing routines (e.g., reading for pleasure five days a week instead of two). Given potential barriers to clinical treatment (e.g., cost, availability of resources) and its focus on ill-being, examining potential activities and practices that can readily be implemented and impact both illbeing and well-being seems to be a fruitful area for research.

\section{The Arts, Humanities, and Flourishing}

A growing domain of research focuses on the connection between arts and humanities engagement and flourishing. Many nations and communities actively track the ways in which its citizens have access to and engage with the arts and humanities, recognizing the important role they play in people's lives (e.g., Cuypers et al., 2012; DiMaggio \& Mukhtar, 2004; Fancourt \& Steptoe, 2019a, 2019b; Fancourt \& Tymoszuk, 2018; Mak et al., 2020; NEA, 2013, 2018; Stern \& Seifert, 2017; Vaananen et al., 2009; Weziak-Bialowolska et al., 2019). For instance, in the United States in $201753.8 \%$ (i.e., 132.3 million) of adults attended an arts event within the past year, an increased attendance rate from prior assessments (NEA, 2018). Other nations, such as the United Kingdom (Fancourt \& Steptoe, 2019a, 2019b; Fancourt \& Tymoszuk, 2019; Tymoszuk et al., 2020), Finland (Vaananen et al., 2009), Sweden (Johansson et al., 2001; Konlaan et al., 2000), Norway (Cuypers et al., 2012), and Poland (Weziak-Bialowolska \& Bialowolski, 2016; WeziakBialowolska et al., 2019), also show notable levels of engagement with the arts and humanities. Further, a recent study with a representative sample of adults in the United States suggests that many adults engage with the arts and humanities on a recurrent basis, most frequently with music and visual art (Crone et al., 2021). Given the existing widespread interest in the arts and 
humanities, understanding the impacts of existing engagement practices on flourishing is a natural next step.

The Positive Humanities are an emerging field that focuses on the relation between the arts and humanities and human flourishing (Pawelski, 2021). The field of the positive humanities has been enormously influenced by the field of positive psychology founded by Martin Seligman. Establishing positive psychology as a "science of positive subjective experience, positive individual traits, and position institutions" (Seligman \& Csikszentmihalyi, 2000, p. 5), Seligman formalized and advocated for a greater emphasis on advancing the understanding and cultivation of greater well-being from a scientific perspective. The Positive Humanities are inspired by the scientific focus of positive psychology on well-being topics and incorporates the approaches used within the humanities to advocate for a balanced and comprehensive approach to human flourishing.

Throughout history, the arts and humanities have been an ever-present force in shaping communities and individuals. Given people's widespread engagement with the arts and humanities, it is vital to understand the impact of this engagement on collective and individual flourishing. Both humanistic (e.g., Kristeller, 1965; Proctor, 1998; Small, 2013) and empirical (e.g., Shim et al., 2019, 2021; Vaziri et al., 2019) scholarship have a considerable history of investigating the flourishing effects of arts and humanities engagement. The Positive Humanities, integrating both approaches, provide a fresh perspective for examining the relation between the arts and humanities and flourishing. In this review, we will focus mainly on the impact of arts and humanities engagement on individual flourishing, not because collective flourishing is any less important but because research on its impact on collective flourishing is significantly less advanced. 
Although the Positive Humanities are an emerging field, research interested in connections between arts and humanities engagement and flourishing has a substantial history. Large-scale studies suggest that greater engagement with the arts and humanities is associated with living longer and lower mortality risks (e.g., Bygren et al., 1996; Fancourt \& Steptoe, 2019b; Konlaan et al., 2000; Vaananen et al., 2009), reduced risk for mental health conditions, such as depression, anxiety, or dementia (e.g., Cuypers et al., 2012; Fancourt et al., 2018; Fancourt \& Steptoe, 2019a; Fancourt \& Tymoszuk, 2018), lower levels of loneliness (e.g., Tymoszuk et al., 2020), higher subjective health (e.g., Cuypers et al., 2012; Weziak-Bialowolska \& Bialowolski, 2016; Wilkinson et al., 2007) and reduced incidence of physical health conditions (e.g., Vaananen et al., 2009). Many of these findings also hold when controlling for relevant demographic factors, such as gender or socioeconomic status ${ }^{2}$. Systematic reviews support the positive association between arts and humanities engagement and flourishing. Both traditional humanities domains (see Vaziri et al., 2019 for review) and a variety of arts disciplines (see Shim et al., 2021 for review) have shown associations between engagement and a range of flourishing outcomes. Similarly, government, agency, and foundation reports on the relationship between arts and humanities engagement and flourishing overwhelmingly support the notion that the arts and humanities have positive implications for people's flourishing (e.g., All-Party Parliamentary Group, 2017; Craemer, 2008; Fujiwara \& MacKerron, 2015; Kilroy et al. 2008; OECD \& ICOM, 2019; McCarthy et al., 2004; Fancourt \& Finn, 2019).

\section{Art Museums and Flourishing}

\footnotetext{
${ }^{2}$ After controlling for demographic variables, the association between attendance and dementia incidence (Fancourt et al., 2018), loneliness (Tymoszuk et al., 2020), and physical health conditions (Vaananen et al., 2009) were reduced. After controlling for gender, only reductions in anxiety and depression remained significant for women; all findings remained significant for men (Cuypers et al., 2012).
} 
More in-depth studies of the relation between arts and humanities engagement and flourishing have helped to clarify the nature of this association. Crone et al. (2021) conducted a survey within a representative United States sample to examine the ways in which people engage with the arts and humanities in their lives. One of the most common domains endorsed (about onethird of the sample) was engagement with the visual arts, and this engagement tended to be in the form of experiencing or appreciating the art rather than producing or creating visual art. Given how common engaging with visual art is, it is important to understand the flourishing impacts of this form of engagement.

\section{Why Art Museums?}

Although visual art may be encountered in a variety of forms (e.g., street art, Mitschke et al., 2017; online, Annechini et al., 2020), art museums remain a common way in which people engage with visual art. When surveyed in 2017 , nearly $25 \%$ of people in the United States reported having visited an art museum or gallery in the previous year (NEA, 2018). Art perception and reactions to art have been long-studied in laboratory contexts (e.g., see Pelowski et al., 2017 for review), but laboratory-based studies do not capture the experience of viewing art in a museum context (e.g., Specker et al., 2017). Accordingly, research is increasingly being conducted within art museums (e.g., Cotter et al., in press; Specker et al., 2017; Rodriguez et al., in press), providing greater insight into art engagement in ecologically-valid contexts. Further, with the increasing interest in the use of art engagement for addressing ill-being and well-being concerns (e.g., AllPart Parliamentary Group, 2017; Craemer, 2008; Fancourt \& Finn, 2019; Kilroy et al., 2008; McCarthy et al., 2004; Sonke et al., 2019), it is necessary to examine the impact of art engagement in the contexts in which it frequently occurs. The museum effect (Smith, 2014) underscores the importance of this point. Research suggests that visitors engage in greater reflection about 
themselves, their future, and broader social concerns over the course of their time in a museum, with these processes peaking in the middle of their visit (Waszkielewicz, 2006). Obviously, this aspect of engagement with art can be studied only in a museum context.

\section{Effects of Art Engagement in Museums}

Given that visiting art museums is a common form of art engagement (NEA, 2013, 2018) and an activity anyone can readily participate in without training, extensive knowledge, or clinician referral, it is unsurprising that researchers have increasingly begun to examine the flourishing effects of different forms of art museum engagement.

Mental and physical health. Several studies have examined the effects of visiting the art museum on clinical and self-report indices of mental and physical health. A common focus has been on the ability of art museum visits to reduce stress. Cortisol, a hormone associated with stress responses, is commonly used as a biological marker of people's stress levels (Clow, 2004). Several studies have observed changes in cortisol after visiting an art museum (Clow \& Fredhoi, 2006; D’Cunha et al., 2019; Grossi et al., 2019). In nonclinical samples, cortisol reductions were observed after a single art museum visit (Clow \& Fredhoi, 2006; Grossi et al., 2019); Clow and Fredhoi (2006) also observed reductions in self-reported stress levels. Researchers have also examined stress responses to art museum visits in people living with dementia and found that repeated art museum engagement, involving both weekly art viewing and art making for 6 weeks, resulted in more dynamic cortisol responses (D’Cunha et al., 2019). Further, engaging in art museum activities has been associated with feeling restored in an elementary school student sample (Annechini et al., 2020) and in decreased emotional exhaustion and depersonalization in medical residents (Orr et al., 2019). 
Beyond examining stress responses and feelings of restoration during art museum visits, researchers have also found associations with other mental health outcomes and physical health. For those most familiar with art museums - art museum staff and frequent museum visitorsvisiting an art museum reduced anxiety levels (Binnie, 2010); however, infrequent museum visitors did not show these anxiety reductions. In people living with dementia, a six-week artmaking and art-viewing program showed reductions in depression symptoms and improvements in cognitive function at the completion of the program (D'Cunha et al., 2019); however, the reductions in depression returned to baseline levels six weeks after the end of the program, whereas cognitive function continued to improve after completion of the program. Further, research suggests that consistently visiting art museums (i.e., every few months) is associated with lower risk of being diagnosed with dementia (Fancourt et al., 2018), even after controlling for demographic characteristics. Additional evidence suggests that more frequent visitation is associated with longer life spans (Konlaan et al., 2000), that viewing figurative art — but not modern art—may reduce blood pressure but not alter heart rate (Mastandrea et al., 2019), and that a one-hour tour for individuals with chronic pain was able to provide self-reported pain relief during the tour and reduced pain intensity three weeks post tour (Koebner et al., 2019).

Subjective well-being and health. In addition to understanding the impacts of art museum visitation on specific physical and mental health factors, researchers have also sought to understand the impacts on global subjective judgments of well-being and health. Subjective judgments of well-being have been linked to visiting the art museum (D'Cunha et al., 2019; Grossi et al., 2019; Michalos, 2005; Michalos \& Kahlke, 2010), and some studies indicate it is the satisfaction with these visits — not frequency — that drives this effect (Michalos, 2005; Michalos \& Kahlke, 2010). Further, art museum visitation is also linked with greater quality of life (Michalos, 
2005; Michalos \& Kahlke, 2010), including in people living with dementia (D'Cunha et al., 2019; Schall et al., 2018), and with levels of self-reported health (Michalos \& Kahlke, 2010; Wilkinson et al., 2007) in the general population.

Emotional well-being. A common focus in art and aesthetics research is the examination of emotional responses to artworks (e.g., Pelowski \& Akiba, 2011; Silvia, 2005). Much of this work emphasizes aesthetic emotions (e.g., Fingerhut \& Prinz, 2020; Menninghaus et al., 2019), but some studies have examined the ability of art museums to influence emotional well-being. Emotional well-being has typically been examined in special populations, including in people living with dementia (Camic et al., 2016; Schall et al., 2018), people with severe mental health conditions (Roberts et al., 2011) and older adults (Herron \& Jamieson, 2020; Thomson et al., 2018). Across these groups, attendance at art-viewing and art-making programming enhanced positive affect (Camic et al., 2016; Roberts et al., 2011; Schall et al., 2018; Thomson et al., 2018). Specifically, art museum engagement has been associated with increased feelings of cheerfulness (Thomson et al., 2018) and happiness (Michalos \& Kahlke, 2010), feeling uplifted and engaged (Herron \& Jamieson, 2020), and viewing time in the art museum as rewarding (Camic et al., 2016).

Social connection and loneliness. Finally, research has examined the role art museum engagement plays in contributing to feeling connected to others. Art museum engagement is associated with greater feelings of the ability to connect with others (Roberts et al., 2011), enhanced social inclusion (Herron \& Jamieson, 2020), and lower feelings of social disconnect (Koebner et al., 2019). For some, visiting the art museum can aid in bolstering bonding social capital (Newman \& McLean, 2004), meaning that art museum engagement allows people to form connections with people with similar interests (Bourdieu, 1997; Putnam, 2000), particularly when the exhibitions have personally relevant foci (e.g., exhibits related to the museum's region; 
Newman \& McLean, 2004). Work on bridging social capital—connecting with individuals with dissimilar backgrounds - is less developed, however, and more research is needed to understand the role of art engagement in cultivating bridging social capital.

Beyond feeling connection with others, satisfaction with art museum visits is associated with viewing art museums as community builders (Michalos \& Kahlke, 2010). Potentially contributing to the view of art museums cultivating connection and community building are the mindset changes visitors undergo over the course of their visit (Smith, 2014). Waszkielewicz (2006) found that during the middle of the visit, compared to the beginning or end of the visit, visitors reported higher levels of reflection on societal topics (e.g., participating in community affairs, concerns for societal issues, contributing to the well-being of others), suggesting that the experience of the visit encourages different forms of reflection and thought processes. Further, it may be possible for art engagement to target specific relational outcomes, such as increased empathy. Sherman and colleagues (2020) found that when visitors were instructed to take the perspective of American Indians depicted in photographic exhibits, visitors showed greater empathy toward American Indians compared to visitors who received no viewing instructions.

\section{Flourishing-Based Arts Programming}

Art museums are purposefully and carefully curated, with institution-specific programs, educational opportunities, and art exhibits. Organizations can play an important role in whether and how flourishing-related outcomes are achieved. Positive deviance in organizations (Spreitzer \& Sonenshein, 2004), for example, represents ways in which organizations depart from the norm in laudable ways. Within an art museum, this may manifest through offering programming aligned with flourishing outcomes or decolonizing collections and exhibition practices to encourage greater diversity in visitor populations. Many institutions are engaging in these practices, such as 
creating specially designed programs for particular populations (e.g., the Meet Me at MoMA program developed for people living with dementia; Rosenberg et al., 2009) or increasing their usage of online offerings to engage a broader audience (e.g., Agostino et al., 2020; Burke et al., 2020).

\section{Art Museums and Flourishing Programs}

One way art museums have sought to address the flourishing of their visitors is through the development and delivery of flourishing-centered programming. A recent survey indicated that nearly $25 \%$ of art museums and galleries in the United Kingdom offer programs focused on flourishing outcomes (All-Party Parliamentary Group, 2017). These programs have tended to be targeted toward specific populations with the aims of addressing particular flourishing outcomes within these populations.

A common focus in museum-based flourishing programs is in enhancing the lives of older adults and adults with dementia. Many of these programs involve both art viewing and art making activities (e.g., Bennington et al., 2016; Roe et al., 2016; Thomson et al., 2018) and target a range of outcomes related to improving emotional well-being (e.g., Thomson et al., 2018) and reducing social isolation (e.g., Dodd \& Jones, 2014; Roe et al., 2016). In these programs, people visit the museum multiple times across several weeks or months and work with trained art educators or art therapists to discuss individual reactions to artworks, relate the works to their personal histories or memories, and engage in art creation in hopes to improve individual flourishing. These programs have shown several benefits to participants, including increasing feelings of social engagement (Bennington et al., 2016; Roe et al., 2016), enhancing positive emotions (Thomson et al., 2018), and overall well-being (Bennington et al., 2016; Thomson et al., 2018). 
In addition to programs geared toward older adults more broadly, there are also numerous programs tailored for people living with dementia with aims to improve flourishing within this population. These programs also target similar outcomes as other types of programs for older adults, including social isolation (Flatt et al., 2015; Rosenberg et al., 2009; MacPherson et al., 2009), emotional well-being (Roseberg et al., 2009, Camic et al., 2016; Schall et al., 2018), and overall quality of life (D’Cunha et al., 2019; Schall et al., 2018). Although some of these programs engage participants in both art viewing and creation activities (e.g., Belver et al., 2018; Camic et al., 2016; Flatt et al., 2015; Schall et al., 2018), several programs for people living with dementia focus on art viewing and discussion (Rosenberg et al., 2009; D’Cunha et al., 2019; MacPherson et al., 2009). As a result of engaging in these programs, participants report higher quality of life (D’Cunha et al., 2019; Schall et al., 2018), greater self-esteem (Flatt et al., 2015; Rosenberg et al., 2009), a greater sense of engagement and social cohesion (Flatt et al., 2015; MacPherson et al., 2009; Schall et al., 2018), and decreases in depression (D’Cunha et al., 2019) and apathy (Schall et al., 2018). Because many people living with dementia reside in specialized care homes and frequently visit medical care settings, participants in these programs especially value their visits to art museums as non-medical settings that can enhance their flourishing (Camic et al., 2016) and as a way to demonstrate their capacity to engage in a variety of activities (MacPherson et al., 2009). The use of a non-medical setting to enhance flourishing in clinical populations is likely an important factor underlying the demonstrated benefits of these types of programs.

\section{Social Prescription of Art}

Although many art museums host their own programs to enhance visitor flourishing, governmental entities have also begun to formalize the usage of art museums and art engagement through social prescribing programs. Social prescribing is an approach that complements and 
operates alongside traditional healthcare systems to improve patient health and to increase positive health behaviors (Alderwick, 2018; Drinkwater et al., 2019). In these systems, people from lower socioeconomic backgrounds, people at risk for social isolation (e.g., older adults), and people with chronic physical or mental health conditions, among others, may need greater support to achieve positive health outcomes. Social prescribing aims to provide greater support to these populations through connecting individuals with resources and activities within their community that can aid in addressing existing health conditions or increasing engagement in positive health related behaviors. In some cases, clinicians will recommend specific activities (e.g., physical exercise within a local park) but in many cases people are referred to link workers who work with individuals to identify activities that are aligned with their interests and that can address the reason for referral. Although such programs have been gaining momentum, the evaluation of these programs has been sparse (Chatterjee et al., 2018).

Social prescribing programs have frequently referred patients to arts activities. Social prescribing of art engagement has most prominently been used in the United Kingdom (All-Party Parliamentary Group, 2017; Potter, 2013) in its 'Arts on Prescription’ program. In this program, people with psychological or physical distress were referred to a variety of community arts activities, including referrals to visit art museums and galleries. Overall, the program was associated with a $37 \%$ decrease in primary care doctor visits and a $27 \%$ reduction in hospital admissions. Although this program was not exclusively focused on the impacts of art museum social prescriptions, art museum prescriptions were a major component in the program. Beyond the 'Arts on Prescription' program, other regions have implemented social prescription of art museum visits, including Montreal (Mercer, 2018; "Montreal museum partners with doctors to 'prescribe' art”, 2018), Brussels (Boffey, 2021), and the United States ("Program opens cultural 
doors to low-income families", 2020; Rhode Island Department of Health, n.d.). Although some social prescriptions of art museums have programming tailored for social prescriptions (e.g., Froggett et al., 2011; Koebner et al., 2018, 2019; Thomson et al., 2018, 2020), in most cases people referred to the art museum are not provided with concrete direction on how to engage with the art museum and are encouraged to explore pre-existing museum programs for things that interest them. However, greater clarity for what people should do when given a prescription to the art museum may enhance the flourishing benefits of this practice. Additionally, because some people receiving social prescriptions may not be frequent visitors to art museums, it is necessary to ensure people are engaged and interested in these experiences in order to experience benefits (O'Neill, 2010). Empirically examining methods to promote greater engagement and interest in visitors and developing best practices for social prescriptions to the art museum are necessary for widespread success of these pratices.

\section{Furthering Art Museums as Institutions for Flourishing}

Collectively, the research on art museums and flourishing suggests that these institutions can have positive impacts on both ill-being and well-being factors. These benefits are seen when people visit the museum of their own volition but also when they take part in flourishing-focused programming. In recognition of these benefits, recent years have seen an uptick in the use of social prescription of art museum visits and found the 'arts on prescription' program to have widereaching benefits for individual flourishing. There are many open questions and topics for future research to address, however.

\section{Examining Mechanisms}

With the consistent findings that art museum engagement is associated with a range of illbeing and well-being outcomes, a logical next step is to explore the mechanisms that underlie these 
associations. The field of psychology has a long history of examining flourishing outcomes and understanding the ways in which to enhance human flourishing. As research progresses in examining the flourishing effects of art museum engagement, it is necessary to examine these existing theories and apply the findings of flourishing research more broadly within art museum contexts. In addition, both the visual arts literature and arts and health literature have recently developed models and frameworks that can serve as a starting point for better understanding these context-specific relationships.

Research on visual art engagement has generated several theoretical models of the processing and understanding of visual art (e.g., Leder et al., 2004; Pelowski et al., 2017; Tinio, 2013). The Vienna Integrated Model of Art Perception (VIMAP; Pelowski et al., 2017), a recently proposed comprehensive theoretical model of responses to visual art, considers both bottom-up and top-down perceptual and cognitive processes during art engagement and proposes five distinct outcomes of these processes: 1) a facile experience in which engagement is relatively superficial; 2) a novel and insightful experience in which people will experience some level of surprise but not deeply engage with the art; 3) a negative experience in which people feel negative emotions and feel the need to disengage with the art; 4) a harmonious experience in which people feel a range of positive states, such as flow, feeling moved, or getting chills, and view the experience as enjoyable; and 5) a transformative experience in which people deeply engage with the art and reflect on their pre-existing schemas and update them based upon their art engagement, typically encountering a range of positive and negative states throughout this process. The latter two outcomes of the VIMAP model-harmonious and transformative experiences - are most closely theoretically aligned with flourishing-relevant outcomes and warrant further investigation to understand the association between type of art experience and flourishing outcomes. 
Additional models and frameworks have also considered how the arts may have specific implications for flourishing outcomes. A recently developed conceptual model (Shim et al., 2019; Tay et al., 2018; Thapa et al., under review) helps to clarify how the nature of arts and humanities engagement contributes to flourishing by identifying five mechanisms through which engagement may lead to flourishing. These mechanisms - Reflection, Acquisition, Immersion, Socialization, and Expression (i.e., the RAISE mechanisms) — provide a useful framing for describing the myriad ways in which people participate in the arts and humanities and why we may observe connections with flourishing. Reflection refers to engaging cognitive and emotional processes during arts and humanities activities that influence our habits, values, or worldview. Acquisition refers to social and cognitive processes of arts and humanities engagement (e.g., mastery experiences) that lead to the development of adaptive and enduring perspectives, habits, and skills (e.g., self-efficacy). Immersion refers to being absorbed in the experience and devoting attention to the cognitive and emotional experiences during art engagement. Socialization refers to how people develop new identities or reaffirm identities relevant to the arts and humanities and influences how people interact with one another. Expression refers to the process of externalizing thoughts and emotions, such as through discussion or via the creation of art. Of these mechanisms, Immersion has been theorized to be a gateway to the others. In order to effectively reflect, acquire skills, socialize, or express yourself, it is necessary to be immersed in the experience (Shim et al., 2019; Tay et al., 2018; Thapa et al., under review). A recent study (Thapa et al., under review) using the RAISE framework has found links between these mechanisms and multiple measures of flourishing, including overall thriving, meaning in life, positive affect, and interpersonal reactivity. The consistent association between these mechanisms and flourishing outcomes suggests the promise of further study involving their rigorous testing via experimental methods. 
One additional perspective (Engh et al., 2021) considers how art engagement is able to foster both individual and community flourishing. The WE Making framework (Engh et al., 2021) emphasizes arts institutions as places able to foster social cohesion, which in turn is believed to influence both individual and community flourishing. This framework suggests that engaging in shared arts experiences within a community can foster greater social cohesion and thus have downstream effects on ill-being and well-being factors. Further, this framework suggests that focusing on social cohesion as one pathway to flourishing may be particularly useful in promoting positive outcomes in communities that experience racism, oppression, or structural disinvestment if additional effort is made to reduce structural barriers (Engh et al., 2021).

Both visual arts-specific theoretical models and conceptual frameworks exploring broad arts engagement provide compelling arguments for why and how the observed relationships between arts engagement and flourishing occur. As the research literature has consistently found links between engagement with art museum visits and flourishing outcomes, the next step is to determine how and why these relationships occur. The models and frameworks discussed here can help guide this next step of research. Testing the mechanisms and claims made by these models will help to advance theory on this topic, provide greater opportunity for experimental studies to examine causality, and will allow for the application of this knowledge to shape people's art engagement in order to optimize flourishing benefits. Researchers may also wish to consult theoretical orientations that consider how leisure activities more broadly, rather than merely artspecific activities, connect to flourishing outcomes and the mechanisms implicated in these relationships (e.g., Fancourt et al., 2021).

\section{Program Development and Evaluation}


With the knowledge that art museum visitation has flourishing-related benefits, there have been increases in the development of museum-based programs (including social prescribing) that aim to reduce ill-being and increase well-being. As more specific theory is developed around the relationship between art museum visits and flourishing, it is necessary to use theory to inform the development of new forms of programming and to rigorously test the efficacy of these programs.

The majority of current art museum programs targeting flourishing have been developed with specific populations of visitors in mind, such as older adults or people living with dementia (e.g., Rosenberg et al., 2009; Thomson et al., 2018). But the broader literature on art engagement and flourishing suggests that positive impacts of art museum visitation is not limited to these populations or other clinical populations. Just as many museums offer programs with educational aims (e.g., tours focused on art historical content), one way to expand the role of art museums as institutions for flourishing is to develop programs with an eye toward flourishing elements. An increasingly common type of program available within art museums involves engaging visitors in meditative or mindfulness practices when viewing art (e.g., Fox, 2020; Friedrich, 2019). Although the flourishing benefits of such programs have not been thoroughly researched, these programs provide a compelling model for future development of flourishing-centered programs for the general population.

In addition to expanding the populations for which flourishing programming is available, it is also necessary to systematically test the effects of these programs. Although there have been a few projects using experimental designs to examine the effects of flourishing programs (e.g., Schall et al., 2018), most evaluations of programs, if any evaluation is completed, use single-group designs that do not allow for strong conclusions regarding the effects of these programs. With the increases in the number of museums hosting these programs and their integration into social 
prescribing programs, it is vital to understand the effects of individual programs in order to make informed recommendations to individuals with social prescriptions to museums and to advocate for effective programs to be brought into other museums.

Although a range of people believe visiting art museums holds the potential to contribute to flourishing (Binnie, 2010; Michalos, 2005; Michalos \& Kahlke, 2010), it is important to note that there may be barriers to achieving these outcomes, such as feeling uncomfortable within the art museum or not being able to connect with the art on exhibit. As new programs and activities are developed for art museums, it is important to ensure programs are accessible and of interest to a wide range of people. One potential option for the creation of more accessible art museum programming or offerings is through development of digital programs or resources, as these reduce barriers related to geographical access or ability to afford admission fees. Such programs have become more prevalent in the wake of the COVID-19 pandemic (e.g., Agostino et al., 2020; Burke et al., 2020) and demonstrate the feasibility of expanding such offerings in the future.

To gain greater understanding of the impacts of particular programs or of art museum visitation, it is necessary to use measures appropriate for capturing flourishing outcomes in museum contexts. Oftentimes, research in art museums has emphasized the measurement of emotional responses (e.g., Pelowski \& Akiba, 2011), art preferences or liking (e.g., Leder \& Nadal, 2014), or visitor learning (e.g., Chisholm et al., 2020); although important factors to understand, inclusion of flourishing outcomes and creation of measures tailored to the museum environment should be a priority. Researchers have begun to create these measures (e.g., Thomson \& Chatterjee, 2015), but they have tended to be limited to emotional experiences and general engagement during a program or museum visit. To further work on flourishing outcomes of specific programs or of unstructured visits to art museums, measures will need to be created that capture a broader variety 
of ill-being and well-being related outcomes and particularly such outcomes that are of particular interest to art museum professionals (e.g., empathy, closed-mindedness; Cotter, 2021). This is a timely recommendation as art museum professionals have identified addressing visitor well-being as an important area for growth for art museums more broadly (Cotter, 2021). Such measures should be developed in partnership with art museums to identify the data that will aid art museums in adapting their practices for flourishing-relevant aims and for connecting outcomes to specific qualities of an art museum visit (e.g., participation in museum programs, engagement with museum resources).

To ensure these programs are effective, there has also been a call for greater integration of art engagement and public health practices. The Creating Healthy Communities: Arts + Public Health in America (Sonke et al., 2019) initiative identifies several ways in which active collaboration between the arts and public health sector can promote flourishing on a larger scale. In their report, the team identifies several recommendations to further these aims, including the need for public health officials and researchers to partner directly with arts organizations to cocreate arts-based interventions and to work together to implement these interventions within arts and culture contexts. In addition to collaborating on intervention development and implementation, it is also necessary for arts organizations and artists to have a voice in how flourishing outcome priorities are determined (Sonke et al., 2019). Following these recommendations, in addition to developing measures geared toward the art museum environment and evaluating the outcomes of flourishing programs, seems a fruitful way forward for flourishing programming in art museums.

\section{Conclusion}

There are many behaviors and activities that people can engage in to benefit their personal flourishing. Visiting art museums, a popular leisure activity (NEA, 2013, 2018; Sharpe \& da Silva, 
2020), is one such activity that has been shown to have positive effects for both ill-being and wellbeing factors at the individual level. Benefits at the community or collective level seem promising, but have yet to be thoroughly examined. Positive individual effects appear to occur both in selfdirected visiting contexts (i.e., under normal conditions of a visit; Grossi et al., 2019; Mastandrea et al. 2018) as well as when participating in specialized programming to benefit a range of flourishing outcomes (e.g., loneliness, quality of life in people with dementia; Roe et al., 2016; Rosenberg et al., 2009).

With the increasing evidence that art museum visitation is linked with flourishing outcomes, it is unsurprising that a variety of governmental agencies and other organizations are seeking to capitalize on these benefits. The development of social prescriptions of arts engagement (e.g., All-Party Parliamentary Group, 2017; Mercer, 2018) represents a major step in recognizing the potential of art museums to contribute to individual flourishing. To fully realize the potential of art museums as institutions for flourishing, it is necessary to develop more specialized instruments for assessing flourishing within art museum contexts and to test theoretically relevant mechanisms that may help in explaining why these associations exist. It is also necessary to develop more effective ways of assessing the flourishing benefits of art museums on communities and society in general. By these means, art museums can be better understood — and advancedas institutions for flourishing. 


\section{References}

Agostino, D., Arnaboldi, M., \& Lampis, A. (2020). Italian state museums during the COVID-19 crisis: From onsite closure to online openness. Museum Management and Curatorship, $35(4), 362-372$.

Alderwick, H. A. J., Gottlieb, L. M., Fichtenberg, C. M., \& Adler, N. E. (2018). Social prescribing in the U. S. and England: Emerging interventions to address patients' social needs. American Journal of Preventative Medicine, 54(5), 715-718.

All-Party Parliamentary Group on Arts, Health, and Wellbeing. (2017). Creative health: The arts for health and wellbeing.

Annechini, C., Menardo, E., Hall, R., \& Pasini, M. (2020). Aesthetic attributes of museum environmental experience: A pilot study with children as visitors. Frontiers in Psychology, 11, 508300.

Belver, M. H., Ullan, A. M., Avila, N., Moreno, C., \& Hernandez, C. (2018). Art museums as a source of well-being for people with dementia: An experience in the Prado Museum. Arts \& Health, 10(3), 213-226.

Bennington, R., Backos, A., Harrison, J., Reader, A. E., \& Carolan, R. (2016). Art therapy in art museums: Promoting social connectedness and psychological well-being of older adults. The Arts in Psychotherapy, 49, 34-43.

Binnie, J. (2010). Does viewing art in the museum reduce anxiety and improve wellbeing? Museums \& Social Issues, 5(2), 191-201.

Boffey, D. (2021, September 2). Brussels doctors to prescribe museum visits for Covid stress. The Guardian. https://www.theguardian.com/world/2021/sep/02/brussels-doctors-toprescribe-museum-visits-for-covid-stress. 
Bourdieu, P. (1997). The forms of capital. In A. H. Halsey, H. Lauder, P. Brown, and A. M. Wells (Eds.). Education, Culture, and Society (pp. 46-58). Oxford: Oxford University Press.

Burke, V., Jorgensen, D., \& Jorgensen, F. A. (2020). Museums at home: Digital initiatives in response to COVID-19. Norsk museumstidsskrift, 6(2), 117-123.

Bygren, L. O., Konlaan, B. B., \& Johansson, S. E. (1996). Attendance at cultural events, reading books or periodicals, and making music or singing in a choir as determinants for survival: Swedish interview survey of living conditions. British Medical Journal, 313, 1577-1580.

Camic, P. M., Baker, E. L., \& Tischler, V. (2016). Theorizing how art gallery interventions impact people with dementia and their caregivers. The Gerontologist, 56(6), 1033-1041.

Chatterjee, H. J., Camic, P. M., Lockyer, B., \& Thomson, L. J. M. (2018). Non-clinical community interventions: A systematised review of social prescribing schemes. Arts \& Health, 10(2), 97-123.

Chisolm, M. S., Kelly-Hedrick, M., Stephens, M. B., \& Zahra, F. S. (2020). Transformative learning in the art museum: A methods review. Family Medicine, 52(10), 736-740.

Close, C., Kouvonen, A., Bosqui, T., Patel, K., O’Reilly, D., \& Donnelly, M. (2016). The mental health and wellbeing of first generation migrants: A systematic-narrative review of reviews. Globalization and Health, 12, 47.

Clow, A. (2004). Cortisol as a biomarker of stress. Journal of Holistic Healthcare, 1(3), 10-14.

Clow, A. \& Fredhoi, C. (2006). Normalisation of salivary cortisol levels and self-report stress by a brief lunchtime visit to an art gallery by London City Workers. Journal of Holistic Healthcare, 3(2), 29-32. 
Cotter, K. N. (2021, September). Well-being aims of art museums: A survey of art museum professionals. Paper presented at the biannual congress of the International Association of Empirical Aesthetics. Online: September 1-3.

Cotter, K. N., Fekete, A., \& Silvia, P. J. (in press). Why do people visit art museums? Examining visitor motivations and visit outcomes. Empirical Studies of the Arts.

Craemer, R. (2008). The arts and health: From economic theory to cost-effectiveness.

Crone, D. L., Tay, L., \& Pawelski, J. O. (2021, July). A large-scale multi-method descriptive study of the content of personally significant arts- and humanities-related experiences and activities. Symposium conducted at the world congress of the International Positive Psychology Association. Online: July 15-17.

Csikszentmihalyi, M. (1990). Flow: The psychology of optimal experience. Harper \& Row.

Cuypers, K., Krokstad, S., Holmen, T. L., Knudtsen, M. S., Bygren, L. O., \& Holmen, J. (2012). Patterns of receptive and creative cultural activities and their association with perceived health, anxiety, depression and satisfaction with life among adults: The HUNT study, Norway. Journal of Epidemiological Community Health, 66, 698-703.

Davidson, R. J. (1998). Affective style and affective disorders: Perspectives from affective neuroscience. Cognition and Emotion, 12, 307-330.

D’Cunha, N. M., McKune, A. J., Isbel, S., Kellett, J., Georgousopoulou, E. N., \& Naumovski, N. (2019). Psychophysiological responses in people living with dementia after an art gallery intervention: An exploratory study. Journal of Alzheimer's Disease, 72(2), 549-562.

Diener, E., Smith, H., \& Fujita, F. (1995). The personality structure of affect. Journal fo Personality and Social Psychology, 69, 130-141. 
Diener, E., Suh, E. M., Lucas, R. E., \& Smith, H. L. (1999). Subjective well-being: Three decades of progress. Psychological Bulletin, 125(2), 276-302.

DiMaggio, P. \& Mukhtar, T. (2004). Arts participation as cultural capital in the United States, 1982-2002: Signs of decline? Poetics, 32, 169-194.

Dodd, J. \& Jones, C. (2014). Mind, body, spirit: How museums impact health and wellbeing.

Drinkwater, C., Wildman, J., \& Moffatt, S. (2019). Social prescribing. The British Medical Journal, 364, I1285.

Duckworth, A. L., Peterson, C., Matthews, M. D., \& Kelly, D. R. (2007). Grit: Perseverance and passion for long-term goals. Journal of Personality and Social Psychology, 92(6), 10871101.

Engh, R., Martin, B., Kidd, S. L., \& Nicodermus, A. G. (2021). WE-making: How arts \& culture unite people to work toward community well-being. Easton, PA: Metris Arts Consulting.

Falk, J. H. (2006). An identity-centered approach to understanding museum learning. Curator: The Museum Journal, 49(2), 151-166.

Falk, J. H. (2008). View art museum visitors through the lens of identity. Visual Arts Research, 34(2), 25-34.

Fancourt, D., Aughterson, H., Finn, S., Walker, E., \& Steptoe, A. (2021). How leisure activities affect health: A narrative review and multi-level theoretical framework of mechanisms of action. Lancet Psychiatry, 8, 329-339.

Fancourt, D. \& Finn, S. (2019). What is the evidence on the role of the arts in improving health and well-being? A scoping review. World Health Organization.

Fancourt, D. \& Steptoe, A. (2019a). Cultural engagement and mental health: Does socioeconomic status explain the association? Social Science \& Medicine, 236, 112425. 
Fancourt, D. \& Steptoe, A. (2019b). The art of life and death: 14 year follow-up analyses of associations between arts engagement and morality in the English Longitudinal Study of Ageing. British Medical Journal, 367, 16377.

Fancourt, D., Steptoe, A., \& Cadar, D. (2018). Cultural engagement and cognitive reserve: Museum attendance and dementia incidence over a 10-year period. British Journal of Psychiatry, 213, 661-663.

Fancourt, D. \& Tymoszuk, U. (2018). Cultural engagement and incident depression in older adults: Evidence from the English Longitudinal Study of Ageing. The British Journal of Psychiatry, 214, 225-229.

Fava, G. A., Ruini, C., Rafanelli, C., Finos, L., Conti, S., \& Grandi, S. (2004). Six-year outcome of cognitive behavior therapy for prevention of recurrent depression. American Journal of Psychiatry, 161, 1872-1876.

Fingerhut, J. \& Prinz, J. J. (2020). Aesthetic emotions reconsidered. The Monist, 103, 223-239.

Flatt, J. D., Liptak, A., Oakley, M. A., Gogan, J., Varner, T., \& Lingler J. H. (2015). Subjective experiences of an art museum engagement activity for persons with early-stage Alzheimer's Disease and their family caregivers. American Journal of Alzheimer's Disease \& Other Dementias, 30(4), 380-389.

Fox, A. (2020, April 22). Unwind with these free, museum-led meditation and mindfulness sessions. Smithsonian Magazine. Retrieved from https://www.smithsonianmag.com/smart-news/unwind-with-free-museum-meditation$\underline{\text { mindfulness-sessions-180974715/. }}$

Fredrickson, B. L. (1998). What good are positive emotions? Review of General Psychology, 2(3), 300-319. 
Friedrich, M. (2019, January 3). The rise of the mindful museum. The Baffler. Retrieved from https://thebaffler.com/latest/the-rise-of-the-mindful-museum-friedrich.

Froggett, L., Farrier, A., Poursanidou, K., Hacking, S., \& Sagan, A. (2011). Who cares?

Museums, health and wellbeing research project: A study of the Renaissance North West Programme.

Fujiwara, D. \& MacKerron, G. (2015). Cultural activities, artforms and wellbeing. Arts Council England.

Gable, S. L. \& Haidt, J. (2005). What (and why) is positive psychology? Review of General Psychology, 9(2), 103-110.

Grossi, E., Blessi, G. T., \& Sacco, P. L. (2019). Magic moments: Determinants of stress relief and subjective wellbeing from visiting a cultural heritage site. Culture, Medicine, and Psychiatry, 43, 4-24.

Herron, A. \& Jamieson, A. (2020). Grandfathers at Melbourne Museum: Shining a spotlight on overlooked museum visitors. Visitor Studies, 23(2), 101-119.

Johansson, S. E., Konlaan, B. B., \& Bygren, L. O. (2001). Sustaining habits of attending cultural events and maintenance of health: A longitudinal study. Health Promotion International, 16(3), 229-234.

Keyes, C. L. M. (2002). The mental health continuum: From languishing to flourishing in life. Journal of Health and Social Research, 43(June), 207-222.

Keyes, C. L. M. (2007). Promoting and protecting mental health as flourishing: A complementary strategy for improving national mental health. American Psychologist, 62(2), 95-108. 
Kilroy, A., Kaagan, C., Garner, C., Parkinson, C., \& Senior, P. (2008). Invest to save: Arts in health evaluation.

Koebner, I. J., Fishman, S. M., Paterniti, D., Sommer, D., Ward, D., \& Joseph, J. G. (2018). Curating care: The design and feasibility of a partnership between an art museum and an academic pain center. Curator, 61(3), 415-429.

Koebner, I. J., Fishman, S. M., Patemiti, D., Sommer, D., Witt, C. M., Ward, D., \& Joseph, J. G. (2019). The art of analgesia: A pilot study of art museum tours to decrease pain and social disconnection among individuals with chronic pain. Pain Medicine, 20(4), 681691.

Konlaan, B. B., Bygren, L. O., \& Johansson, S. E. (2000). Visiting the cinema, concerts, museums or art exhibitions as determinant of survival: A Swedish fourteen-year cohort follow-up. Scandinavian Journal of Public Health, 28, 174-178.

Kristeller, P. O. (1965). Renaissance thought II: Papers on humanism and the arts. New York: Harper \& Row.

Leder, H., Belke, B., Oeberst, A., \& Augustin, D. (2004). A model of aesthetic appreciation and aesthetic judgments. British Journal of Psychology, 95(4), 489-508.

Leder, H. \& Nadal, M. (2014). Ten years of a model of aesthetic appreciation and aesthetic judgments: The aesthetic episode—Developments and challenges in empirical aesthetics. British Journal of Psychology, 105(4), 443-464.

MacPherson, S., Bird, M., Anderson, K., Davis, T., \& Blair, A. (2009). An art gallery access programme for people with dementia: 'You do it for the moment'. Aging \& Mental Health, 13(5), 744-752. 
Mak, H. W., Coulter, R., \& Fancourt, D. (2020). Does arts and cultural engagement vary geographically? Evidence from the UK household longitudinal study. Public Health, 185, 119-126.

Mastandrea, S., Maricchiolo, F., Carrus, G., Giovannelli, I., Giuliani, V., \& Berardi, D. (2019). Visits to figurative art museums may lower blood pressure and stress. Arts \& Health, $11(2), 1-10$.

McCarthy, K. F., Ondaatje, E. H., Zakaras, L., \& Brooks, A. (2004). Gifts of the muse: Reframing the debate about the benefits of the arts.

Menninghaus, W., Wagner, V., Wassiliwizky, E., Schindler, I., Hanich, J., Jacobsen, T., \& Koelsch, S. (2019). What are aesthetic emotions? Psychological Review, 126(2), 171195.

Mercer, C. (2018). Primary care providers exploring value of "social prescriptions" for patients. CMAJ, 10(190), E1463-4.

Michalos, A. C. (2005). Arts and the quality of life: An exploratory study. Social Indicators Research, 71, 11-59.

Michalos, A. C. \& Kahlke, P. M. (2010). Arts and perceived quality of life in British Colombia. Social Indicators Research, 96, 1-39.

Mitschke, V., Goller, J., \& Leder, H. (2017). Exploring everyday encounters with street art using a multimethod design. Psychology of Aesthetics, Creativity, and the Arts, 11(3), 276-283.

Montreal museum partners with doctors to 'prescribe' art. (2018, October 26). BBC. https://www.bbc.com/news/world-us-canada-45972348.

National Endowment for the Arts. (2013). How a nation engages with art: Highlights from the 2012 survey of public participation in the arts. 
National Endowment for the Arts. (2018). U.S. trends in arts attendance and literary reading: 2002-2017. A first look at results from the 2017 survey of public participation in the arts.

Newman, A. \& McLean, F. (2004). Capital and the evaluation of the museum experience. International Journal of Cultural Studies, 7(4), 480-498.

Niemiec, R. M. (2013). VIA character strengths: Research and practice (The first 10 years). In Well-being and cultures (pp. 11-29). Springer, Dordrecht.

Oishi, S. \& Westgate, E. C. (2021). A psychologically rich life: Beyond happiness and meaning. Psychological Review.

O'Neill, M. (2010). Cultural attendance and public mental health—-from research to practice. Journal of Public Mental Health, 9(4), 22-29.

Organisation for Economic Co-Operation and Development \& International Council of Museums (2019). Culture and local development-: Maximising the impact. Guide for local governments, communities and museums.

Orr, A. R., Moghbeli, N., Swain, A., Bassett, B., Niepold, S., Rizzo, A., \& DeLIsser, H. M. (2019). The fostering resilience through art in medical education (FRAME) workshop: A partnership with the Philadelphia Museum of Art. Advances in Medical Education and Practice, 10, 361-369.

Pawelski, J. O. (2013). What is the eudaimonic turn? In J. O. Pawelski \& D. J. Moores (Eds.), The eudaimonic turn: Well-being in literary studies (pp. 1-26). Madison, NJ: Fairleigh Dickinson University Press.

Pawelski, J. O. (2016a). Defining the 'positive' in positive psychology: Part II. A normative analysis. Journal of Positive Psychology, 11(4), 357-365. 
Pawelski, J. O. (2016b). Bringing together the humanities and the science of well-being to advance human flourishing. In D. Harward (Ed.), Well-being and higher education: A strategy for change and the realization of education's greater purposes (pp. 207-216). Washington, DC: Bringing Theory to Practice.

Pawelski, J. O. (2021). The positive humanities: Culture and human flourishing. In L. Tay and J. O. Pawelski (Eds.), The Oxford Handbook of the Positive Humanities.

Pekarik, A. J. \& Schreiber, J. B. (2012). The power of expectation. Curator: The Museum Journal, 55(4), 487-496.

Pelowski, M. \& Akiba, F. (2011). A model of art perception, evaluation and emotion in transformative aesthetic experience. New Ideas in Psychology, 29(2), 80-97.

Pelowski, M., Markey, P. S., Forster, M., Gerger, G., \& Leder, H. (2017). Move me, astonish me...delight my eyes and brain: The Vienna Integrated Model of top-down and bottomup processes in Art Perception (VIMAP) and corresponding affective, evaluative, and neurophysiological correlates. (2017). Physics of Life Review, 21, 80-125.

Peterson, C. \& Seligman, M. E. P. (2004). Character strengths and virtues: A handbook of classification (Vol. 1). Oxford University Press.

Phelan, S., Bauer, J., \& Lewalter, D. (2018). Visit motivations: Development of a short scale for comparison across sites. Museum Management and Curatorship, 33(1), 25-41.

Potter, S. (2013). Arts on prescription 2010-12.

Prilleltensky, I., Dietz, S., Prilleltensky, O., Myers, N. D., Rubenstein, C. L., Jin, Y., \& McMahon, A. (2015). Assessing multidimensional well-being: Development and validation of the I COPPE scale. Journal of Community Psychology, 43(2), 199-226. 
Proctor, R. E. (1998). Defining the humanities: How rediscovering a tradition can improve our schools ( $2^{\text {nd }}$ ed.). Bloomington: Indiana University Press.

Program opens cultural doors to low-income families. (2020, January 18). Daily Hampshire Gazette. https://www.gazettenet.com/New-program-that-gives-cheaper-or-free-access-tocultural-attractions-across-the-state-launched-in-Amherst-32080622.

Putnam, R. (2000). Bowling alone: The collapse and revival of American community. New York: Simon and Schuster.

Rhode Island Department of Health (n.d.). Arts and health. Retrieved September 7, 2021 from https://health.ri.gov/healthcare/about/artsandhealth/.

Roberts, S., Camic, P. M., \& Springham, N. (2011). New roles for art galleries: Art-viewing as a community intervention for family carers of people with mental health problems. Arts \& Health, 3(2), 146-159.

Rodriguez, R. M., Fekete, A., Silvia, P. J., \& Cotter, K. N. (in press). The art of feeling different: Exploring the diversity of emotions experienced during an art museum visit. Psychology of Aesthetics, Creativity, and the Arts.

Roe, B., McCormick, S., Lucas, T., Galagher, W., Winn, A., \& Elkin, S. (2016). Coffee, cake \& culture: Evaluation of an art for health programme for older people in the community. Demenia, 15(4), 539-559.

Rosenberg, F., Parsa, A., Humble, L., \& McGee, C. (2009). Meet me: Making art accessible to people with dementia.

Russell, J. A. \& Carroll, J. M. (1999). On the bipolarity of positive and negative affect. Psychological Bulletin, 125, 3-30. 
Ryan, R. M. \& Deci, E. L. (2000). Self-determination theory and the facilitation of intrinsic motivation, social development, and well-being. American Psychologist, 55, 68-78.

Ryff, C. D. (1995). Psychological well-being in adult life. Current Directions in Psychological Science, 4, 99-104.

Ryff, C. D., Love, G. D., Urry, H. L., Muller, D., Rosenkranz, M. A., Friedman, E. M., Davidson, R. J., \& Singer, B. (2006). Psychological well-being and ill-being: Do they have distinct or mirrored biological correlates? Psychotherapy and Psychosomatics, 75, 85-95.

Ryff, C. D. \& Singer, B. (1998). The contours of positive human health. Psychological Inquiry, 9, 1-28.

Sargent, C., Gebruers, C., \& O’Mahony, J. (2017). A review of the physiological and psychological health and wellbeing of naval service personnel and the modalities used for monitoring. Military Medical Research, 4, 1.

Schall, A., Tesky, V. A., Adams, A. K., \& Pantel, J. (2018). Art museum-based intervention to promote emotional well-being and improve quality of life in people with dementia: The ARTEMIS project. Dementia, 17(6), 728-743.

Seligman, M. E. P. (in press). Foreword. In L. Tay and J. O. Pawelski (Eds.) The Oxford Handbook of the Positive Humanities.

Seligman, M. E. P. (2012). Flourish: A visionary new understanding of happiness and wellbeing. Simon and Schuster.

Seligman, M. E. P. \& Csikszentmihalyi, M. (2000). Positive psychology: An introduction. American Psychologist, 55(1), 5-14. 
Sharpe, E. \& da Silva, J. (2020). Ai Wewei is world's most popular artist. The Art Newspaper, 29(322), 2-3.

Sherman, A., Cupo, L., \& Mithlo, N. M. (2020). Perspective-taking increases emotionality and empathy but does not reduce harmful biases against American Indians: Converging evidence from the museum and lab. PLoS ONE, 15(2), e0228784.

Shim, Y., Jebb, A. T., Tay, L., \& Pawelski, J. O. (2021). Arts and humanities interventions for flourishing in healthy adults: A mixed studies systematic review. Review of General Psychology, 25(3), 258-282.

Shim, Y., Tay., L., Ward, M., \& Pawelski, J. O. (2019). The arts and humanities: An integrative conceptual framework for psychological research. Review of General Psychology, 23, $159-176$.

Silvia, P. J. (2005). Emotional responses to art: From collation and arousal to cognition and emotion. Review of General Psychology, 9(4), 342-357.

Small, H. (2013). The value of the humanities. Oxford: Oxford University Press.

Smith, J. K. (2014). The museum effect: How museums, libraries, and cultural institutions educate and civilize society. Rowman \& Littlefield.

Sonke, J., Golden, T., Francois, S., Hand, J., Chandra, A., Clemmons, L., Fakunle, D., Jackson, M. R., Magsmen, S., Rubin, V., Sams, K., Springs, S., \& Steinmetz, M. P. (2019). Creating health communities through cross-sector collaboration.

Specker, E., Tinio, P. P. L., \& van Elk, M. (2017). Do you see what I see? An investigation of the aesthetic experience in the laboratory and museum. Psychology of Aesthetics, Creativity, and the Arts, 11(3), 265-275. 
Spreitzer, G. M \& Sonenshein, S. (2004). Toward the construct definition of positive deviance. American Behavioral Scientist, 47(6), 828-847.

Stern, M. J. \& Seifert, S. C. (2017). The social wellbeing of New York City's neighborhoods: The contribution of culture and the arts.

Tay, L., Pawelski, J. O., \& Keith, M. G. (2018). The role of the arts and humanities in human flourishing: A conceptual mode. Journal of Positive Psychology, 13(3), 215-225.

Thapa, S., Vaziri, H., Shim, Y., Tay, L., \& Pawelski, J. O. (under review). Development and validation of the mechanisms of engagement in the arts and humanities scales.

Thomson, L. J. \& Chatterjee, H. J. (2015). Measuring the impact of museum activities on wellbeing: Developing the museum well-being measures toolkit. Museum Management and Curatorship, 30(1), 44-62.

Thomson, L. J., Lockyer, B., Camic, P. M., \& Chatterjee, H. J. (2018). Effects of a museumbased social prescription intervention on quantitative measures of psychological wellbeing in older adults. Perspectives in Public Health, 138(1), 28-38.

Thomson, L. J., Morse, N., Elsden, E., \& Chatterjee, H. J. (2020). Art, nature and mental health: Assessing the biopsychosocial effects of a 'creative green prescription' museum programme involving horticulture, artmaking and collections. Perspectives in Public Health, 140(5), 277-285.

Tinio, P. P. L. (2013). From artistic creation to aesthetic reception: The mirror model of art. Psychology of Aesthetics, Creativity, and the Arts, 7(3), 265-275.

Tymoszuk, U., Perkins, R., Fancourt, D., \& Williamon, A. (2020). Cross-sectional and longitudinal association between receptive arts engagement and loneliness among older adults. Social Psychiatry and Psychiatric Epidemiology, 55, 891-900. 
Urry, H. Ll., Nitschke, J. B., Dolski, I., Jackson, D. C., Dalton, K. M., Mueller, C. J., Rosenkranz, M. A., Ryff, C. D., Singer, B. H., \& Davidson, R. J. (2004). Making a life worth living: Neural correlates of well-being. Psychological Science, 15, 367-372.

Vaananen, A., Murray, M., Koskinen, A., Vahtera, J., Kouvonen, A., \& Kivimaki, M. (2009). Engagement in cultural activities and cause-specific mortality: Prospective cohort study. Preventive Medicine, 49, 142-147.

VanderWeele, T. J. (2017). On the promotion of human flourishing. Proceedings of the National Academy of Sciences, 114(31), 8148-8156.

Vaziri, H., Tay, L., Keith, M. G., \& Pawelski, J. O. (2019). History, literature, and philosophy: A systematic review of positive functioning. Journal of Positive Psychology, 14(6), 695723.

Waszkielewicz, I. I. (2006). Encounters with art: The psychological impact of art museum visitation [Unpublished doctoral dissertation]. Rutgers University.

Weziak-Bialowolska, D. \& Bialowolski, P. (2016). Cultural events—Does attendance improve health? Evidence from a Polish longitudinal study. BMC Public Health, 16, 730.

Weziak-Bialowolska, D., Bialowolski, P., \& Sacco, P. L. (2019). Involvement with the arts and participation in cultural events-Does personality moderate impact on well-being? Evidence from the U. K. household panel survey. Psychology of Aesthetics, Creativity, and the Arts, 13(3), 348-358.

Wilkinson, A. V., Waters, A. J., Bygren, L. O., \& Tarlov, A. R. (2007). Are variation in rates of attending cultural activities associated with population health in the United States. BMC Public Health, 7, 226. 
World Health Organization (1948). World Health Organization constitution. In Basic documents.

Geneva: Author. 